Pacific Journal of Mathematics

ON THE ZEROS OF DERIVATIVES OF BALANCED 


\title{
ON THE ZEROS OF DERIVATIVES OF BALANCED TRIGONOMETRIC POLYNOMIALS
}

\author{
CARL L. Prather
}

\begin{abstract}
The final set and initial set for a balanced $2 \pi$-periodic trigonometric polynomial with roots in a horizontal strip are obtained. Assuming the limits have finite order, we show that only trigonometric polynomials are uniform limits on compact sets of balanced $2 \pi$-periodic trigonometric polynomials that have only real zeros. The final set result is extended to balanced almost periodic trigonometric polynomials. Finally, we give a final set result for balanced exponential sums whose exponents are complex.
\end{abstract}

1. Introduction. The well known Gauss-Lucas theorem for algebraic polynomials states that the zeros of the derivative $P^{\prime}(z)$ of a complex valued polynomial $P(z)$ lie in the convex hull of the zeros of $P(z)$.

Let $T(z)$ be a trigonometric polynomial having the form

$$
T(z)=\sum_{k=-N}^{+N} a_{k} \exp (i k z)
$$

where $a_{-N} a_{N} \neq 0$. Such trigonometric polynomials are called balanced by Genchev ([3], [4], [5]). He proves the following analogues of the Gauss-Lucas theorem for balanced trigonometric polynomials:

(1) If $T(z)$ has its zeros in the strip $a \leqq \operatorname{Im} z \leqq b$, then all the zeros of $T^{\prime}(z)$ also lie in this strip.

(2) If $T(z)$ has zeros in the strip $a \leqq \operatorname{Im} z \leqq b$, then all the zeros of the trigonometric polynomial

$$
L(T)(z)=\int_{z-i \lambda}^{z+i \lambda} T(t) d t, \lambda>0,
$$

also lie in the strip [3].

Repeated applications of Genchev's result in (1) shows that the zeros of all the successive derivatives $T^{(n)}(z), n=1,2,3, \cdots$ of a balanced trigonometric polynomial likewise have their zeros in this strip.

Furthermore, these zeros must accumulate somewhere, since each $T^{(n)}(z)$ is $2 \pi$-periodic. The set of accumulation points of all the zeros of the successive derivatives form what we call the final set [11, page 197]. Included in the final set are points where infinitely many derivatives vanish.

We apply the same idea to Genchev's result referred to in (2), 
by letting $L^{n}(T)(z)$ denote the composition of the operator $L$ with itself $n$ times. We determine the points of accumulation of the zeros of $L^{n}(T)(z)$, for $n=0,1,2, \ldots$. We shall call this set of accumulation points the initial set. We determine the final and initial sets in these cases.

THEOREM 1.1. Given $T(z)=\sum_{k=-N}^{+N} a_{k} \exp (i k z)$, where $a_{-N} a_{N} \neq 0$, whose zeros lie in the strip $a \leqq \operatorname{Im} z \leqq b$, the final set consists of the zeros of the function $a_{-N} \exp (-i N z)+a_{N} \exp (i N z)$ together with the zeros of its derivative. That is, the final set consists of the discrete set of points

$$
z=(-i / 2 N) \log \left(a_{-N} / a_{N}\right)+(1 / 2 N) \arg \left( \pm a_{-N} / a_{N}\right)+\pi t / N \text { for } t
$$

an integer and these points all lie on the horizontal line $\operatorname{Im} z=$ $(-1 / 2 N) \log \left|a_{-N} / a_{N}\right|$.

Proof. The $n$th derivative of $T(z)$ is given by $T^{(n)}(z)=$ $\sum_{k=-N}^{+N} i^{n} k^{n} a_{k} \exp (i k z)$, which is equal to $i^{n} N^{n}\left[(-1)^{n} a_{-N} \exp (-i N z)+\right.$ $\left.a_{N} \exp (i N z)\right]+i^{n} \sum_{k=-N+1}^{N-1} k^{n} a_{k} \exp (i k z)$. We consider the sequence of functions $H_{n}(z)=T^{(n)}(z) / i^{n} N^{n}$. This is equal to $\left[(-1)^{n} a_{-N}\right.$ $\left.\exp (-i N z)+a_{N} \exp (i N z)\right]+\left(1 / N^{n}\right) \sum_{k=-N+1}^{N-1} k^{n} a_{k} \exp (i k z)$. Now as $n \rightarrow \infty$, the sum $\left(1 / N^{n}\right) \sum_{k=-N+1}^{N-1} k^{n} a_{k} \exp (i k z) \rightarrow 0$ uniformly on compact sets. When $n$ is odd (resp. even) the first term is $-a_{-N}$ $\exp (-i N z)+a_{N} \exp (i N z)\left(\operatorname{resp} . a_{-N} \exp (-i N z)+a_{N} \exp (i N z)\right)$. The sequence $\left\{H_{n}(z)\right\}$ for $n$ odd is such that $H_{n}(z) \rightarrow-a_{-N} \exp (-i N z)+$ $a_{N} \exp (i N z)$ as $n \rightarrow \infty$ uniformly on compact sets. By Hurwitz's theorem the zeros of $-a_{-N} \exp (-i N z)+a_{N} \exp (i N z)$ are points of accumulation of the zeros of these $H_{n}(z)$ and hence of the zeros of the corresponding $T^{(n)}(z)$. Similarly, for $n$ even, the sequence $\left\{H_{n}(z)\right\}$ is such that $H_{n}(z) \rightarrow a_{-N} \exp (-i N z)+a_{N} \exp (i N z)$ as $n \rightarrow \infty$ uniformly on compact sets. Again by Hurwitz's theorem, the zeros of $a_{-N} \exp (i N z)+a_{N} \exp (i N z)$ are points of accumulation of the zeros of $T^{(n)}(z)$ ( $n$ even). Now, the zeros $-a_{-N} \exp (-i N z)+a_{N}$ $\exp (+i N z)$ consists of the points $z=(-i / 2 N) \log \left|a_{-N} / a_{N}\right|+(1 / 2 N)$ $\arg \left(a_{-N} / a_{N}\right)+\pi t / N$, for $t$ any integer, and the zeros of $a_{-N}$ $\exp (-i N z)+a_{N} \exp (+i N z)$ consists of the points $z=(-i / 2 N)$ $\log \left|a_{-N} / a_{N}\right|+(1 / 2 N) \arg \left(-a_{-N} / a_{N}\right)+\pi t / N$, for $t$ any integer.

Thus the final set of $T$ does consist of that discrete set of points, which is contained in the line $\operatorname{Im} z=(-1 / 2 N) \log \left|a_{-N} / a_{N}\right|$. This end the proof of Theorem 1.1.

REMARK. The discrete points of the final set are precisely the accumulation points of the zeros of the successive derivatives of a balanced trigonometric polynomial. For if we are given the 
zeros of $\pm a_{-N} \exp (-i N z)+a_{N} \exp (i N z), a_{-N} a_{N} \neq 0$, then $\sum_{k=-N}^{+N} a_{k}$ $\exp (i k z)$ has as its final set this collection of zeros.

Furthermore, since the imaginary part of the final set lies between $a$ and $b$, we have

$$
\begin{aligned}
a \leqq & (-1 / 2 N) \log \left|a_{-N} / a_{N}\right| \leqq b, \\
& -2 N a \geqq \log \left|a_{-N} / a_{N}\right| \geqq-2 N b,
\end{aligned}
$$

and so

$$
1 / \exp (2 N a) \geqq\left|a_{-N} / a_{N}\right| \geqq 1 / \exp (2 N b) .
$$

As a special case of the above theorem, note that when $\left|a_{-N}\right|=$ $\left|a_{N}\right|$, the zeros of the successive derivatives of $T(z)$ condense to discrete points on the real axis.

THEOREM 1.2. Given $T(z)=\sum_{k=-N}^{+N} a_{k} \exp (i k z)$ a balanced trigonometric polynomial whose zeros lie in the strip $a \leqq \operatorname{Im} z \leqq b$, then the initial set consists of the discrete set of points $z=(-i / 2 N)$ $\log \left|a_{-N} / a_{N}\right|+(1 / 2 N) \arg \left(-a_{-N} / a_{N}\right)+\pi t / N$ ( $t$ any integer) all of which lie on the horizontal line $\operatorname{Im} z=(-1 / 2 N) \log \left|a_{-N} / a_{N}\right|$.

REMARK. Hence for balanced trigonometric polynomials with zeros in $a \leqq \operatorname{Im} z \leqq b$, the initial set is a subset of the final set.

Proof. We start with $T(z)=\sum_{k=-N}^{+N} a_{k} \exp (i k z)=a_{0}+\sum_{k=-N}^{++N} a_{k}$ $\exp (i k z)$, where the prime $\left({ }^{\prime}\right)$ indicates that the zeroth term is omitted. We get that $L(T)(z)=\sum_{k=-N}^{+N}\left(a_{k} / i k\right)[\exp (-k \lambda)-\exp (k \lambda)]$ $\exp (i k z)+2 i \lambda a_{0}$. An elementary induction shows that

$$
\left(L^{n} T\right)(z)=\sum_{k=1}^{N}[S(k)]^{n}\left\{a_{-k} e^{-i k z}+a_{k} e^{i k z}\right\}+(2 i \lambda)^{n} a_{0}
$$

where $S(x)=2 \sinh (\lambda x) / i x$. Since $(\sinh (\lambda x)) / x$ increases as $x \rightarrow \infty$ for fixed $\lambda>0$, it follows that $L^{n}(T)(z) /(S(N))^{n}$ converges uniformly on compact sets to $a_{-N} \exp (-i N z)+a_{N} \exp (i N z)$.

By Hurwuitz's theorem the zeros of that last function are points of accumulation of the zeros of $L^{n}(T)(z) /(S(N))^{n}$, and hence of the zeros of $L^{n}(T)(z)$. As we saw earlier, the zeros of $a_{-N} \exp (-i N z)+$ $a_{N} \exp (i N z)$ are the discrete points $z=(-i / 2 N) \log \left|a_{-N} / a_{N}\right|+$ $(1 / 2 N) \arg \left(-a_{-N} / a_{N}\right)+\pi t / N, t$ any integer.

These are a discrete set of points which lie on the horizontal line $\operatorname{Im} z=(-1 / 2 N) \log \left|a_{-N} / a_{N}\right|$. When $\left|a_{-N}\right|=\left|a_{N}\right|$, the initial set consists of the discrete set of real numbers $(1 / 2 N) \arg \left(-a_{-N} / a_{N}\right)+$ $\pi t / N$ for $t$ any integer. This ends the proof of Theorem 1.2.

2. Approximation by balanced trigonometric polynomials. 
It is well known that the only entire functions that are uniform limits on compact sets of algebraic polynomials whose zeros are real are functions of the classical Laguerre-Pólya class that have the form $f(z)=A z^{m} \exp \left(-\alpha z^{2}+\beta z\right) \pi_{k=1}^{\infty} E\left(z / \alpha_{k}, 1\right)$, where $\alpha \geqq 0, \beta$ and the $\alpha_{k}$ are real and $\sum_{k=1}^{\infty} 1 /\left(\alpha_{k}\right)^{2}<\infty$ [9]. Since $f(z)=\lim _{n \rightarrow \infty} P_{n}(z)$, where the polynomials $P_{n}(z)$ have only real zeros, we have $f^{(k)}(z)=$ $\lim _{n \rightarrow \infty} P_{n}^{(k)}(z)$. By Rolle's theorem, the $P_{n}^{(k)}(z)$ likewise only have real zeros.

Theorem 1 of Genchev says that if the zeros of a balanced trigonometric polynomial $T(z)$ are real, then the zeros of the successive derivatives $T^{(k)}(z), k=1,2, \cdots$ are also real. It is then natural to ask "What are the uniform limits on compact sets of balanced $2 \pi$-periodic trigonometric polynomials whose zeros are real?" We partially answer this by the next result.

THEOREM 2.1. The only entire functions $f \not \equiv$ of finite order that can be approximated uniformly on compact sets by balanced $2 \pi$-periodic trigonometric polynomials having only real zeros are themselves trigonometric polynomials.

Lemma 1. The only entire functions of finite order that are periodic with no real zeros are functions of the form $\exp (a z+b)$.

Proof. Since $f$ is of finite order without zeros, $f(z)=\exp (g(z))$ for $g$ a polynomial by the Hadamard factorization theorem. Then as $\exp (g(z+a))=\exp (g(z))$, we get $\exp (g(z+a)-g(z))=1 \Rightarrow g(z+a)-$ $g(z)=2 \pi i K$ for some integer $K \Rightarrow g^{\prime}(z+a)=g^{\prime}(z)$. So $g^{\prime}$ is a periodic polynomial $\Rightarrow g^{\prime}(z)$ is constant $\Rightarrow g(z)$ is linear.

Proof of Theorem 2.1. The limit function $f$ is $2 \pi$-periodic. Therefore the counting function $n(r)$ of the zeros is $0(1)$ and $\sum_{\left|a_{n}\right| \leqq r} 1 / a_{n}=0(1)$ as $r \rightarrow \infty$, since the positive and negative zeros $\left\{a_{n}\right\}$ are "essentially" symmetric (a shift of the origin to the beginning of a $2 \pi$-period makes them symmetric). Lindelöf's theorem [1, page 27] implies that the canonical product for the $\left\{a_{n}\right\}$ is of exponential type. Since $f$ and $P$ are periodic, so is $e^{g}$ and since $f$ is of finite order, $g$ is a polynomial. By Lemma 1, $g$ is linear. Hence $f$ is of exponential type. A well known theorem [1, page 109] gives that $f$ must be a trigonometric polynomial.

\section{REMARKS.}

(A) Presumably the assumption of finite order in this theorem can be removed and the result will remain valid. 
(B) Let $\left\{T_{n}(z)\right\}_{n=1}^{\infty}$ be a sequence of balanced trigonometric polynomials that converges uniformly on compact sets to $f$. Since $T_{n}$ and all its derivatives have only real zeros, the same is true for $f$ and its derivatives. If $f$ is real entire, then a result of Hellerstein and Williamson [8] implies that $f$ is in the Laguerre-Pólya class and in particular of finite order. Theorem 2.1 then implies that $f$ is a trigonometric polynomial. If, however, $f$ is not a real entire function, then if $f$ is assumed to have finite order, as in the hypothesis of Theorem 2.1, it follows from a theorem of Alander (reproved in the paper of Hellerstein and Williamson) and the periodicity of $f$ that $f$ is a trigonometric polynomial of the form $f(z)=a\left(e^{i c z}-e^{i d}\right)$, $a$ arbitrary, $b, c$, and $d$ real.

3. Some Gauss-Lucas results. We now prove a Gauss-Lucas result for any canonical product of genus 1 having zeros in any horizontal strip.

THEOREM 3.1. Let $P(z)$ be a canonical product of genus 1 whose zeros lie in $|\operatorname{Im} z|<A$. Then the zeros of $P^{\prime}(z)$ also lie in $|\operatorname{Im} z|<A$.

REMARK. Proving the stated theorem suffices to prove this result when the zeros lie in any horizontal strip, since by a change of variable we can always vertically translate any horizontal strip so that it is symmetric about the real axis.

Proof. We will prove this in a series of steps, by demonstrating the following two facts:

(I) Given $P(z)=\pi_{k=1}^{\infty} E\left(z / \alpha_{k}, 1\right)$, where $\alpha_{k}=\psi_{k}+i \tau_{k}$ and the $\alpha_{k}$ satisfy $0<b \leqq \tau_{k} \leqq c$, then for $y>c$, it is true that $\operatorname{Im}\left[f^{\prime} \mid f\right]<0$.

(II) Given $P(z)$ as in (I) and the $\alpha_{k}$ satisfy $b \leqq \tau_{k} \leqq c<0$, then for $y<b$, it is true that $\operatorname{Im}\left[f^{\prime} / f\right]>0$.

Claim. It suffices to prove (I) and (II) to prove this Theorem. For if $P(z)$ is given as above $P(z-i A)$ has zeros in $0<\operatorname{Im} z<2 A$ and by (I) $P^{\prime}(z-i A) \neq 0$ for $y \geqq 2 A$. Similarly, $P(z+i A)$ has zeros in $-2 A<\operatorname{Im} z<0$ and by (II) $P^{\prime}(z+i A) \neq 0$ for $y \leqq-2 A$. But the zeros of $P^{\prime}(z)$ are just vertical translations of the zeros of $P^{\prime}(z-i A)$ and $P^{\prime}(z+i A)$. Therefore, if the zeros of $P(z)$ lie in $|\operatorname{Im} z|<A$, so do the zeros of $P^{\prime}(z)$ lie in $|\operatorname{Im} z|<A$.

Now for the proofs of (I) and (II): We let $\alpha_{k}=\psi_{k}+i \tau_{k}$ and $z=x+i y$. Then an easy computation shows that

$$
\operatorname{Im}\left[P^{\prime}(z) / P(z)\right]=\sum_{k=1}^{\infty}\left\{\left(\tau_{k}-y\right) /\left[\left(x-\psi_{k}\right)^{2}+\left(y-\tau_{k}\right)^{2}\right]-\tau_{k} /\left(\psi_{k}^{2}+\tau_{k}^{2}\right)\right\} .
$$


If as in (I) $0<b \leqq \tau_{k} \leqq c$ and $y>c$, then $\tau_{k}-y<0$ and $-\tau_{k}<0$ so that $\operatorname{Im}\left[f^{\prime} / f\right]<0$. If as in (II), $B \leqq \tau_{k} \leqq c<0$ and $y<b$, then $\tau_{k}-y>0$ and $-\tau_{k}>0$ so that $\operatorname{Im}\left[f^{\prime} / f\right]>0$. This completes the proof.

It is now possible to give an alternate proof of Genchev's theorem for almost periodic trigonometric polynomials. For some basic properties of general almost periodic trigonometric polynomials, see [2] or [8, page 246].

DEFINITION. We say that an almost periodic trigonometric polynomial $T(z)=\sum_{k=-N}^{+N} c_{k} \exp \left(i \lambda_{k} z\right)$ is balanced if the exponents $\lambda_{k}$ are contained in the symmetric interval $[-D,+D]$ with $c_{-N} c_{N} \neq 0$ and where $\lambda_{-N}=-D$ and $\lambda_{N}=+D$.

THEOREM 3.2. Let $T(z)=\sum_{K=-N}^{+N} c_{k} \exp \left(i \lambda_{k} z\right)$ be a balanced almost periodic sum whose roots lie in a horizontal strip $|\operatorname{Im} z| \leqq M$. Then the roots of $T^{\prime}(z)$ also lie in $|\operatorname{Im} z| \leqq M$.

REMARK. Note that this also includes Genchev's theorem for the derivative operator, since a $2 \pi$-periodic balanced trigonometric polynomial is clearly almost periodic.

Proof. By definition, we have that the $\lambda_{k}$ lie in $[-D,+D]$, with $\pm D$ occurring. It is a fact that $f(z)$ is an entire function of exponential type $D$ by $[8$, Theorem 1 and its corollary, pages 266-268]. Moreover, it is true that $[-i D,+i D]$ is its indicator diagram [1, Corollary 6.94, page 108]. We therefore know that the indicator diagram has length $2 D$. This, by definition, makes $f$ a function of the class $[D]$ (see [8, page 211]). B. Ja. Levin and M. G. Krein characterized functions of the class $[D]$ by the following theorem [8, Appendix 6, Theorem 4, page 453]: $T(z)$ is an almost periodic function of the class $[D] \leftrightarrow 1) T(z)$ can be expressed in the form $T(z)=c \lim _{N \rightarrow \infty} \prod_{K=-N}^{+N}\left(1-z / \alpha_{k}\right)$, where $c$ is a constant. There are two other properties that $T$ satisfies in this characterization. Since they won't be used, we omit them.

Hence by (1) $T(z)=c \lim _{N \rightarrow \infty} \Pi_{-N}^{+N}\left(1-z / \alpha_{k}\right)$ as $N \rightarrow \infty$; so $T^{\prime}(z)=$ $c \lim _{N \rightarrow \infty}\left[\Pi_{-N}^{+N}\left(1-z / \alpha_{k}\right)\right]^{\prime}$ and since $\prod_{k=-N}^{+N}\left(1-z / \alpha_{k}\right)$ has its roots in $|\operatorname{Im} z| \leqq M$, by hypothesis, by Theorem 3.1, the zeros of its derivative $\left[\prod_{k=-N}^{+N}\left(1-z / \alpha_{k}\right)\right]^{\prime}$ also lie in $|\operatorname{Im} z| \leqq M$. By Hurwitz's theorem, $T^{\prime}(z)$ also has its roots in $|\operatorname{Im} z| \leqq M$.

REMARK. Theorem 3.2 is true when the zeros lie in any horizontal strip $a \leqq \operatorname{Im} z \leqq b$, since Theorem 3.1 is true in this case. 
(4) A final set result. Now that Genchev's theorem has been demonstrated for (finite sums of) almost periodic balanced trigonometric polynomials, we also have established a final set result for this class of polynomials analogous to Theorem 1.1.

THEOREM 4.1. Given $T(z)=\sum_{k=-N}^{+N} a_{k} \exp \left(i \lambda_{k} z\right), \quad a$ balanced almost periodic trigonometric polynomial whose zeros lie in $a \leqq$ $\operatorname{Im} z \leqq b$, with $\min \left[\lambda_{k}\right]_{K=-N}^{+N}=\lambda_{-N}=-D$ and $\max \left[\lambda_{k}\right]_{k=-N}^{+N}=\lambda_{N}=+D$, then the final set consists of the zeros of the function $a_{-N}$ $\exp (-i D z)+a_{N} \exp (i D z)$ together with the zeros of its derivative. That is, the final set consists of the discrete set of points.

$z=(-i / 2 D) \log \left|a_{-N} / a_{N}\right|+(1 / 2 D)$ arg $\left( \pm a_{-N} / a_{N}\right)+\pi t / D(t$ any integer).

The proof of this is similar to the proof of Theorem 1.1.

(5) Final sets for balanced exponential sums. We determine the final set for balanced finite exponential sums whose exponents are complex.

DEFINITION. The exponential sum $T(z)=\sum_{k=1}^{n} a_{k} \exp \left(\lambda_{k} z\right)$, where $\lambda_{k} \in C$ and the conjugate indicator diagram $I$ of $T$ (i.e., the convex hull of $\left.\left\{\lambda_{1}, \cdots, \lambda_{n}\right\}\right)$ is a polygon where the exponents $\neq 0$, is said to be balanced when with $\Delta=\max _{1 \leqq k \leqq n}\left|\lambda_{k}\right|$, the circle $|z|=\Delta$ contains 2 or more vertices of $I$ and the coefficients corresponding to these vertices are nonzero.

THEOREM 5.1. Let $T(z)=\sum_{k=1}^{n} a_{k} \exp \left(\lambda_{k} z\right)$ be balanced with $|z|=$ $\Delta$ containing $\lambda_{1}, \cdots, \lambda_{p}$, for $1<p \leqq n$ and $a_{1}, \cdots, a_{p}$ all nonzero. Then the final set for $T$ consists of the roots of either finitely many trigonometric sums (which happens when any two $\arg \left(\lambda_{k}\right)$ differ by a rational multiple of $\pi$ for all $k=1, \cdots, p$ ) or limits of roots of infinitely many (which happens when some two arg $\left(\lambda_{k}\right)$ differ by an irrational multiple of $\pi$. Each sum contains terms corresponding to one of the factors $\exp \left(\lambda_{k} z\right), k=1, \cdots, p$.

Proof. Since $\left|\lambda_{1}\right|=\cdots=\left|\lambda_{p}\right|$, let $\lambda_{k}=e^{i \theta k} \lambda_{1}$ for $k=1, \cdots, p$ (with $\theta_{1}=0$ ). Let $z$ belong to a compact set. Then

$$
\begin{aligned}
& T^{(m)}(z) /\left(\lambda_{1}\right)^{m}=\sum_{k=1}^{p} a_{k} e^{i m \theta_{k}} \exp \left(\lambda_{k} z\right)+\left(1 /\left(\lambda_{1}\right)^{m}\right) \sum_{k=p+1}^{n}\left(\lambda_{k}\right)^{m} \exp \left(\lambda_{k} z\right) \\
& =\sum_{k=1}^{p} a_{k} e^{i m \theta_{k}} \exp \left(\lambda_{k} z\right)+(\text { term which converges to } 0 \text { in } \\
& \quad \text { modulus as } m \rightarrow \infty) .
\end{aligned}
$$

By Hurwitz's theorem, when $\theta_{k}$ is a rational multiple of $\pi$, for $k=$ 
$1, \cdots, p$, the final set consists of the roots of finitely many trigonometric sums $\sum_{k=1}^{p} a_{k} e^{i m \theta_{k}} \exp \left(\lambda_{k} z\right)$ as $m \rightarrow \infty^{1}$. Otherwise, the final set consists of limits of roots of infinitely many $\sum_{k=1}^{p} a_{k} e^{i m \theta_{k}} \exp \left(\lambda_{k} z\right)$, as $m \rightarrow \infty$.

The roots of each such sum were investigated by Pólya [10] and Schwengeler [12]. They show that the roots of each such sum are asymptotic to the rays which emanate from the origin and are parallel to the normals to the sides of $\bar{I}$, the indicator diagram of $I$ (in other words, the convex hull of $\left\{\bar{\lambda}_{1}, \cdots, \bar{\lambda}_{p}\right\}$, where $\bar{\lambda}_{j}$ denotes the conjugate of $\lambda_{j}$. By asymptotic to is meant that for any sectors containing any these rays of angle $\varepsilon$, for any $\varepsilon>0$, all but finitely many zeros of $T^{(m)}$ lie in these sectors.

REMARK. For the case when $|z|=\Delta$ contains only two vertices $\lambda_{1}$ and $\lambda_{k}$, say, and $\arg \left(\lambda_{k}\right)-\arg \left(\lambda_{1}\right)$ is an irrational multiple of $\pi$, then the final set for the balanced trigonometric sum $\sum_{k=1}^{n} a_{k}$ $\exp \left(\lambda_{k} z\right)$ consists of the roots of infinitely many sums of the form $a_{k} e^{i m \theta_{k}} \exp \left(\lambda_{k} z\right)+a_{1} e^{i m \theta_{1}} \exp \left(\lambda_{1} z\right)$ as $m \rightarrow \infty$. The roots of these infinitely many sums are dense along the rays that they lie on. It would be interesting to know if this happens in general, that is, when $|z|=\Delta=\max _{1 \leqq k \leqq n}\left|\lambda_{k}\right|$ contains more than 2 vertices.

\section{REFERENCES}

1. R. P. Boas, Jr., Entire Functions, Academic Press, New York, 1954.

2. H. Bohr, Almost Periodic Functions, Chelsea Publishing Co., New York, 1947.

3. T. Genchev, Inequalities for entire functions of exponential type, Proc. Amer. Math. Soc., 56 (1976), 183-188.

4. - A generalization of $S$. N. Bernstein's inequality for trigonometric polynomials, Sov. Math. Dokl., 16, No. 4 (1975), 806-810.

5. ——A Gauss Lucas theorem on trigonometric polynomials, Comp. Rend. de l'Acad. Bulgare des Sci. Tome 28 No. 4 (1975), 449-451.

6. S. Hellerstein and J. Williamson, Derivatives of entire functions and a question of Pólya, Trans. Amer. Math. Soc., 227 (1977), 227-249.

7. - Derivatives of entire functions and a question of Polya, Bul. Amer. Math. Soc., 81 (1975), No. 2, 453-455.

8. B. Ja. Levin, On the distribution of the zeros of entire functions, Translations of Math. Monographs, Vol. 5 Amer. Math. Soc., Providence, R. I., (1964).

9. G. Pólya, Uber Annäherung durch Polynome mit lauter reeler Wurzeln from George Polya: Collected Works, Location of Zeros, R. P. Boas, Jr. editor, M. I. T. Press, Cambridge, Mass., 1974, Paper [16], (1913), 54-70.

10. — Geometrisches über die Verteilung der Nullstellen gewisser ganzer transzendenter Functioner, Ibid., Paper [59], (1920), 198-203.

11. - On the zeros of the derivatives of an analytic function and its analytic character, Ibid., Paper [167], (1943), 394-407.

12. E. Schwengeler, Geometrisches über die Verteilung der Nullstellen spezieller ganzer Funktioner (Exponential-summer), Zurich Dissertation, 1925.

${ }^{1}$ Since $\exp \left(i m \theta_{k}\right)$ is periodic in $m$. 
Received November 21, 1977 and in revised form October 17, 1978. This paper is an abridged version of part of a dissertation written at Northwestern University under the direction of Professor R. P. Boas, Jr.

VPI AND STATE UNIVERSITY

BLACKSBURG, VA 24061 



\title{
PACIFIC JOURNAL OF MATHEMATICS
}

\section{EDITORS}

\author{
DONALD BABBITT (Managing Editor) \\ University of California \\ Los Angeles, CA 90024 \\ HUGo ROSSI \\ University of Utah \\ Salt Lake City, UT 84112 \\ C. C. MOORE \\ University of California \\ Berkeley, CA 94720
}

J. DugundJI

Department of Mathematics

University of Southern California

Los Angeles, CA 90007

R. FinN and J. Milgram

Stanford University

Stanford, CA 94305

\section{ASSOCIATE EDITORS}
E. F. BECKENBACH
B. H. Neumann
F. WolF
K. YOSHIDA

\section{SUPPORTING INSTITUTIONS}

\author{
UNIVERSITY OF BRITISH COLUMBIA \\ CALIFORNIA INSTITUTE OF TECHNOLOGY \\ UNIVERSITY OF CALIFORNIA \\ MONTANA STATE UNIVERSITY \\ UNIVERSITY OF NEVADA, RENO \\ NEW MEXICO STATE UNIVERSITY \\ OREGON STATE UNIVERSITY \\ UNIVERSITY OF OREGON
}

UNIVERSITY OF SOUTHERN CALIFORNIA

STANFORD UNIVERSITY

UNIVERSITY OF HAWAII

UNIVERSITY OF TOKYO

UNIVERSITY OF UTAH

WASHINGTON STATE UNIVERSITY

UNIVERSITY OF WASHINGTON

The Supporting Institutions listed above contribute to the cost of publication of this Journal, but they are not owners or publishers and have no responsibility for its content or policies.

Mathematical papers intended for publication in the Pacific Journal of Mathematics should be in typed form or offset-reproduced, (not dittoed), double spaced with large margins. Please do not use built up fractions in the text of the manuscript. However, you may use them in the displayed equations. Underline Greek letters in red, German in green, and script in blue. The first paragraph or two must be capable of being used separately as a synopsis of the entire paper. Please propose a heading for the odd numbered pages of less than 35 characters. Manuscripts, in triplicate, may be sent to any one of the editors. Please classify according to the scheme of Math. Reviews, Index to Vol. 39. Supply name and address of author to whom proofs should be sent. All other communications should be addressed to the managing editor, or Elaine Barth, University of California, Los Angeles, California, 90024.

50 reprints to each author are provided free for each article, only if page charges have been substantially paid. Additional copies may be obtained at cost in multiples of 50 .

The Pacific Journal of Mathematics is issued monthly as of January 1966. Regular subscription rate: $\$ 72.00$ a year (6 Vols., 12 issues). Special rate: $\$ 36.00$ a year to individual members of supporting institutions.

Subscriptions, orders for numbers issued in the last three calendar years, and changes of address should be sent to Pacific Journal of Mathematics, P.O. Box 969, Carmel Valley, CA 93924, U.S.A. Older back numbers obtainable from Kraus Periodicals Co., Route 100, Millwood, NY 10546.

PUBLISHED BY PACIFIC JOURNAL OF MATHEMATICS, A NON-PROFIT CORPORATION

Printed at Kokusai Bunken Insatsusha (International Academic Printing Co., Ltd.). 8-8, 3-chome, Takadanobaba, Shinjuku-ku, Tokyo 160, Japan.

Copyright (C) 1979 by Pacific Journal of Mathematics Manufactured and first issued in Japan 


\section{Pacific Journal of Mathematics}

\section{Vol. 81, No. $2 \quad$ December, 1979}

Ersan Akyildiz, Vector fields and equivariant bundles ............... 283

Ehrhard Behrends, The centralizer of tensor products of Banach spaces ( $a$ function space representation) ......................... 291

Geoffrey R. Burton, Congruent sections of a convex body ............. 303

John Warnock Carlson, $H$-closed and countably compact extensions ...... 317

Robert Charles Carlson, Eigenfunction expansions for selfadjoint

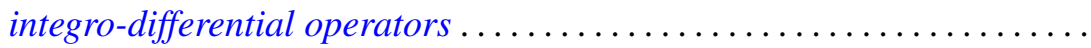

Robert Damiano, Coflat rings and modules.

Eric Karel van Douwen and Washek (Vaclav) Frantisek Pfeffer, Some properties of the Sorgenfrey line and related spaces ................

Uri Elias, Necessary conditions and sufficient conditions for disfocality and

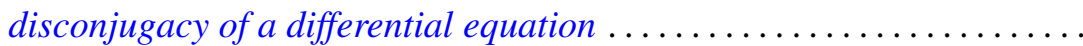

V. L. (Vagn Lundsgaard) Hansen, Polynomial covering spaces and homomorphisms into the braid groups .......................

Paul Hess, Dedekind's problem: monotone Boolean functions on the lattice

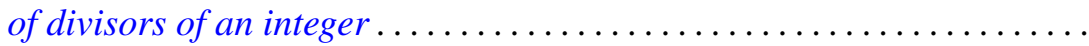

Alan Hopenwasser and David Royal Larson, The carrier space of a reflexive

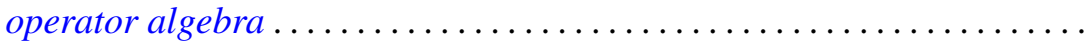

Kyung Bai Lee, Spaces in which compacta are uniformly regular $G_{\delta} \ldots \ldots$.

Claude Levesque, A class of fundamental units and some classes of

Jacobi-Perron algorithms in pure cubic fields...

Teck Cheong Lim, A constructive proof of the infinite version of the

Belluce-Kirk theorem ..........................

Dorothy Maharam and A. H. Stone, Borel boxes

Roger McCann, Asymptotically stable dynamical systems are linear

Peter A. McCoy, Approximation and harmonic continuation of axially symmetric potentials in $E^{3} \ldots \ldots \ldots \ldots \ldots \ldots \ldots$

Takahiko Nakazi, Extended weak-* Dirichlet algebras ....

Carl L. Prather, On the zeros of derivatives of balanced trigonometric polynomials ................................

Iain Raeburn, An implicit function theorem in Banach spaces...

Louis Jackson Ratliff, Jr., Two theorems on the prime divisors of zeros in completions of local domains...

Gloria Jean Tashjian, Cartesian-closed coreflective subcategories of

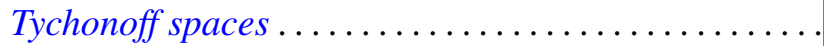

Stephen Edwin Wilson, Operators over regular maps.... . . 